\title{
Panorama nacional del embarazo precoz en México: lecciones aprendidas en un sexenio
}

\author{
Pablo Kuri-Morales, ${ }^{1}$ Rosalinda Guevara-Guzmán, ${ }^{2}$ Vivian Phillips-Gutiérrez, ${ }^{2}$ Anayeli Mota-Sánchez ${ }^{2}$ y \\ Claudia Díaz-Olavarrieta²* \\ ${ }^{1}$ Academia Nacional de Medicina de México; ²Universidad Nacional Autónoma de México, Facultad de Medicina, División de Investigación. Ciudad \\ de México, México
}

\section{Resumen}

En el mundo, el embarazo adolescente constituye un grave problema de salud pública de índole multifactorial. Específicamente para la mujer conlleva diversas implicaciones educativas, económicas y sociales que afectan su proyecto de vida y amplían las brechas sociales en este grupo etario. Asimismo, las adolescentes son más vulnerables por el riesgo en salud que implica un embarazo a corta edad; según la Organización Mundial de la Salud, "la probabilidad de muerte materna es dos veces más en las adolescentes respecto a las mujeres que se encuentran entre los 20 y 30 años de edad, y para las menores de 15 años los riesgos son cinco veces mayores". En general, las y los adolescentes experimentan una gran necesidad de educación en temas sobre salud sexual y reproductiva, la cual debe dirigirse al aumento de información y conocimientos sobre el uso correcto y acceso a métodos anticonceptivos modernos, así como a desmitificar los temores y creencias en torno a sus posibles efectos secundarios. Asegurar una consejería adecuada y con personal capacitado es igualmente vital. Las instituciones públicas tienen la responsabilidad social de respaldar los esfuerzos encaminados a prevenir el embarazo adolescente, con base en las líneas de acción y políticas sanitarias.

PALABRAS CLAVE: Embarazo adolescente. Salud sexual. Políticas de salud pública. México.

\section{National panorama of adolescent pregnancy in Mexico: lessons learned in a six-year period}

\begin{abstract}
Globally, adolescent pregnancy constitutes a serious public health problem of a multifactorial nature. Specifically for women, it entails various educational, economic and social implications that affect their life project and widen the social gaps in this age group. Furthermore, adolescent girls are more vulnerable because of the health risk involved with pregnancy at a younger age. According to the World Health Organization, "the probability of maternal death is twice as high in adolescents in comparison with women aged between 20 and 30 years, and for those younger than 15 years, the risks are 5 times higher." In general, adolescents are in great need for education on sexual and reproductive health issues, which should be aimed at increasing information and knowledge about correct use and access to modern contraceptive methods, as well as at demystifying fears and beliefs around their possible side effects. Ensuring proper counseling with trained personnel is equally vital. Public institutions have a social responsibility to support efforts aimed at preventing adolescent pregnancy, based on relevant lines of action and health policies.
\end{abstract}

KEY WORDS: Adolescent pregnancy. Sexual health. Public health policies. Mexico. 


\section{Introducción}

De acuerdo con cifras de la Organización Mundial de la Salud, aproximadamente 16 millones de adolescentes de 15 a 19 años y un millón de niñas menores de 15 años dan a luz cada año, ${ }^{1}$ la mayoría en países como México, considerado por el Banco Mundial con una economía de ingresos medianos, y en países con altos índices de pobreza y marginación. ${ }^{2}$

En el orbe, las complicaciones durante el embarazo y el parto son la segunda causa de muerte entre adolescentes; anualmente, alrededor de tres millones de jóvenes con embarazos no deseados se someten a abortos inseguros, practicados en condiciones de riesgo por personal no capacitado. ${ }^{3}$ Si bien desde 1990 se ha registrado un descenso considerable en las tasas de fecundidad adolescente en todo el mundo, esta reducción ha sido irregular y se estima que todavía $11 \%$ de todos los nacimientos se presenta en niñas o adolescentes, de las cuales $95 \%$ vive en países con índices de desarrollo humano medio y bajo. En 2014, la tasa promedio mundial de fecundidad entre adolescentes de 15 a 19 años fue de 49 por cada 1000.

Según estimaciones del Consejo Nacional de Población (Conapo), para 2015 y 2019 la tasa específica de fecundidad (TEF, definida como el número de nacimientos por cada mil mujeres en un grupo etario) en adolescentes de 15 a 19 años correspondió al indicador internacional comprometido por México en el marco de los Objetivos de Desarrollo Sostenible ${ }^{4}$ y ante el Consenso de Montevideo. ${ }^{5}$ En 2015, la TEF en las adolescentes de 15 a 19 años fue de 74.3 y se estima que en el recuento final para 2019 sea de 69.5. La fuente de dicha información proviene de las estimaciones preliminares del Conapo con base en las Proyecciones de la Población 2016-2050. ${ }^{6}$ Las estimaciones de fertilidad adolescente para las niñas de 10 a 14 años constituye un reto metodológico ya que la medida que se utiliza no es una tasa, sino una razón. Lo anterior debido a que se asume que la mayoría de las niñas de 10 y 11 años aún no presentan su primera menstruación (denominador), por lo que no pueden considerarse como expuestas al riesgo de embarazo.

La razón y la tasa de fecundidad en niñas y adolescentes de 10 a 14 años y de 12 a 14 años, aprobadas en el seno del Grupo Interinstitucional de Prevención de Embarazo Adolescente, ${ }^{7}$ para dar seguimiento a la magnitud de nacimientos en menores de 15 años, no son cifras que se utilicen en forma oficial sino como mecanismo de seguimiento y evaluación de la Estrategia Nacional de Prevención de Embarazo Adolescente. ${ }^{8}$ Los esfuerzos de prevención e intervención llevados a cabo por las distintas instancias gubernamentales son vastos e innovadores, sin embargo, el embarazo adolescente es un fenómeno en el que se intersecan variables complejas y que frecuentemente no responden a estrategias exitosas que se han implementado para combatir otros problemas de salud.

La epidemia de embarazo adolescente se ha estudiado de manera fragmentada, como una consecuencia de la inequidad de género que afecta adversamente a las niñas y adolescentes que viven en comunidades rurales o áreas metropolitanas periurbanas de alta marginación. ${ }^{9}$ La epidemia de embarazo adolescente es el resultado de diversos problemas, entre ellos:

- De la calidad de la educación que se oferta a los jóvenes, que no les permite diseñar un plan de vida ambicioso en el que se posponga la reproducción para edades más tardías; ${ }^{10}$

- De un entorno sociocultural en el que la maternidad es vista como un valor que supera logros académicos, laborales o personales; ${ }^{11}$

- De la falta de acceso real a los métodos anticonceptivos que las jóvenes puedan usar y a conocimiento basado en evidencia, incluidos los métodos reversibles de larga duración de última generación diseñados para nulíparas. ${ }^{12}$

- De la transmisión intergeneracional de la pobreza, en la que los patrones de conducta se replican especialmente de madres a hijas; ${ }^{13}$

- De coerción reproductiva,${ }^{14}$ otra manifestación de la violencia basada en el género, y el acoso o violencia sexual que sufren las jóvenes cotidianamente al interior de sus hogares y en su entorno, ${ }^{15}$ que limita su capacidad para negociar el sexo seguro.

- Del hecho que a menor edad reproductiva, menor espaciamiento de los hijos y mayor número de parejas que apoyen a las mujeres a afrontar el sustento familiar. ${ }^{16}$

Entre las consecuencias biológicas y sociales del embarazo adolescente encontramos las siguientes:

- Tasa de mortalidad más elevada durante el parto y puerperio, ${ }^{17}$ como resultado de la desnutrición, obesidad o sobrepeso antes, durante y después del parto. ${ }^{18}$

- Hijos con bajo peso al nacimiento. ${ }^{19}$ 


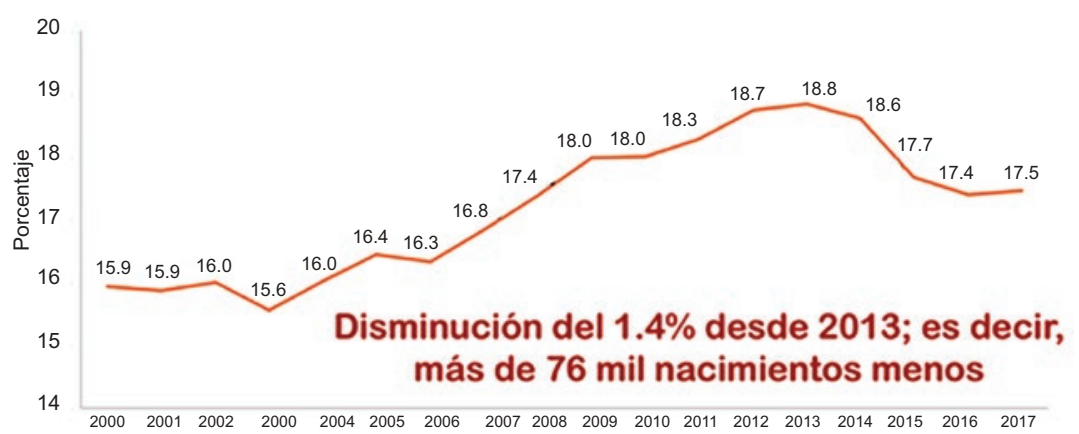

Figura 1. Porcentaje de nacimientos en madres niñas y adolescentes (menores de 20 años), México, 2000-2017, Fuente: INEGI. Nacimientos por año de registro según edad de la madre al nacimiento, incluye madres con residencia habitual en el extranjero, 2019.

- Incremento en el número de hogares liderados por mujeres, ${ }^{20}$ que históricamente son más pobres.

- Analfabetismo, más común en las mujeres. ${ }^{21}$

- Uso explosivo de las redes sociales entre las jóvenes, que presenta una imagen aspiracional que difícilmente encuentra una traducción objetiva en su cotidianidad. ${ }^{22}$

A lo anterior se suma la falta de voluntad política de algunos grupos y al conservadurismo de los poderes fácticos, ${ }^{23}$ los cuales impiden que las jóvenes y mujeres puedan ejercer su derecho constitucional a una vida libre de violencia, ${ }^{24}$ al acceso a una amplia gama de métodos de planificación familiar ${ }^{25}$ que el personal de salud debidamente capacitado oferte en servicios amigables para jóvenes, ${ }^{26}$ incluida la interrupción voluntaria del embarazo (IVE) dentro de las primeras 20 semanas de gestación cuando el embarazo es producto de violación o violencia sexual, o a la interrupción del embarazo dentro de las primeras 12 semanas por otros motivos, procedimiento que se despenalizó en la Ciudad de México en abril del 2007 y que puede realizarse en forma legal, segura y gratuita en los servicios de salud de la Secretaría de Salud de la Ciudad de México. 25,27

Desde 2003, el acceso al aborto legal y seguro es una prioridad de la Organización Mundial de la Salud. ${ }^{3,28}$ La región de América Latina y el Caribe cuenta con la legislación más restrictiva sobre aborto, ${ }^{29}$ incluso comparada con la legislación vigente en África subsahariana. El embarazo adolescente es producto de los determinantes sociales, culturales, educativos e históricos mencionados y tiene consecuencias no exploradas sistemáticamente, como el riesgo a la salud del binomio madre-hijo, al inicio de la vida y al cursar la vida adulta. ${ }^{30}$ Otra asociación importante es la que vincula el embarazo temprano con el estatus metabólico de las madres, ya que se ha identificado elevada incidencia de síndrome metabólico en mujeres mexicanas y los reportes sugieren que el embarazo precoz incrementa este riesgo. ${ }^{31}$

\section{Situación mundial y el caso de México}

El embarazo en niñas y adolescentes, problema que afecta a prácticamente todo el mundo, también ocasiona más de 70000 muertes por complicaciones asociadas al embarazo y al parto, además de que se estima que ocurren al menos 3.2 millones de abortos no seguros. ${ }^{32}$

Tan solo en México, en 2017 se registraron más de 2.2 millones de nacimientos; $17.5 \%$ ocurrió en mujeres de entre 10 y 19 años, es decir, un poco más de 390000 , de los cuales 9748 fueron productos de la concepción en niñas de entre 10 y 14 años. ${ }^{33}$

\section{Respuesta institucional}

Ante este panorama, la Organización Mundial de la Salud ha propuesto diversas estrategias para enfrentar el problema, las cuales incluyen la limitación del matrimonio antes de los 18 años, el incremento de la oferta de métodos de planificación familiar, la reducción del aborto inseguro y el aumento de la atención calificada antes durante y después del parto. ${ }^{34}$

En México, en enero de 2015 se lanzó la Estrategia Nacional para la Prevención del Embarazo en Adolescentes, cuyo objetivo principal es reducir el número de embarazos en adolescentes en México, con absoluto respeto a los derechos humanos, particularmente los derechos sexuales y reproductivos, y alcanzar para 2030 dos metas medibles: disminuir a cero los nacimientos en niñas de 10 a 14 años y reducir en $50 \%$ la tasa específica de fecundidad en adolescentes de 15 a 19 años. En las Figuras 1 a 3 se muestra la tendencia de nacimientos en madres niñas y adolescentes durante el periodo 2000-2017. 


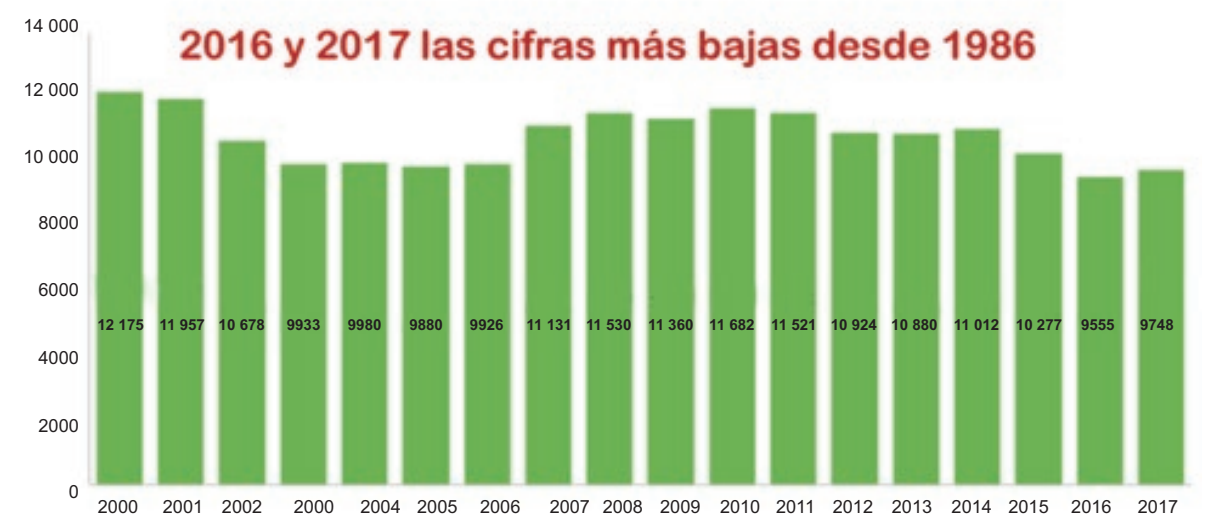

Figura 2. Nacimientos de madres menores de 15 años, México, 2000-2017. Fuente: INEGI. Nacimientos por año de registro según edad de la madre al nacimiento, incluye madres con residencia habitual en el extranjero, 2019.

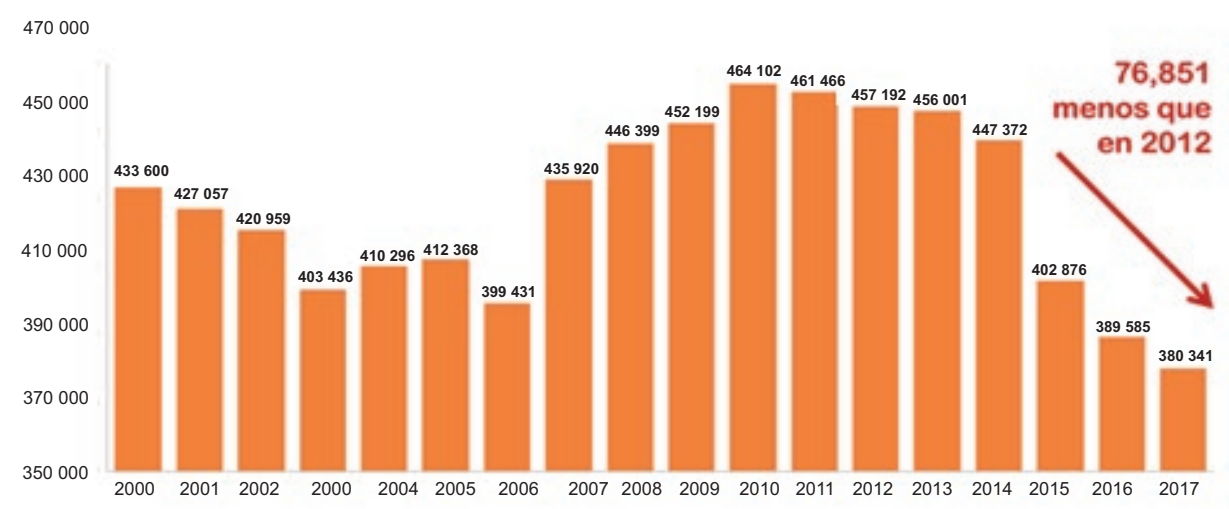

Figura 3. Nacimientos de madres de 15 a 19 años, México, 2000-2017. Fuente: INEGI. Nacimientos por año de registro según edad de la madre al nacimiento, incluye madres con residencia habitual en el extranjero, 2019.

Se encomendó al Consejo Nacional de Población (Conapo) la tarea de coordinar la estrategia, en la que participaron diversas entidades gubernamentales: las secretarías de Salud, de Educación Pública, de Desarrollo Social, el Instituto Nacional de las Mujeres y las instituciones de seguridad social.

Se propusieron diversas acciones, entre las que destacan la creación del Grupo Interinstitucional de Prevención del Embarazo en Adolescentes (como elemento central de coordinación técnica), la capacitación de los recursos humanos que tuvieron la responsabilidad de hacer la traducción operativa de la estrategia, el fortalecimiento de los Servicios Amigables para Adolescentes, la compra centralizada de anticonceptivos, la focalización de las acciones, la difusión y evaluación de la estrategia. También se elaboró y publicó el Modelo de Atención Integral a la Salud Sexual y Reproductiva para Adolescentes (MAISSRA). A continuación se describen algunas de las acciones mencionadas:

\section{Capacitación}

Se llevaron a cabo diversas actividades presenciales y a distancia para capacitar a personal, fundamentalmente de salud, en materia de aplicación del MAISSRA y de la Norma oficial mexicana NOM-047-SSA2-2015, para la atención a la salud del grupo de 10 a 19 años de edad, tarea complicada por la multiplicidad de actores y participantes.

\section{Servicios amigables para adolescentes}

En los últimos seis años, estos servicios se incrementaron en $146.9 \%$. Para diciembre de 2018 se contaba con 2605; $83.6 \%$ del total de municipios del país cuenta con al menos un servicio amigable; en 2017 y 2018 se atendieron anualmente más de 900 mil adolescentes en los servicios amigables, entre dos y tres atenciones al año por adolescente. 
Se ha observado una afluencia importante de menores de 15 años y de hombres adolescentes; los servicios más demandados son la orientación-consejería, la prevención y atención de infecciones de transmisión sexual y la prescripción de anticonceptivos. ${ }^{35}$

Debe destacarse que estos servicios son atendidos por médicos, enfermeras o psicólogos, pero es muy relevante la presencia de jóvenes capacitados que apoyan la operación de los servicios amigables.

\section{Compra de anticonceptivos}

Se tomó la decisión de centralizar las compras de insumos de planificación familiar, en virtud de que algunos estados no los adquirían. Entre 2014 y 2018 se invirtieron más de 4000 millones de pesos para tal fin y si bien no necesariamente se alcanzaron todas las unidades de salud con todos los métodos que se adquirieron, se incrementó la oferta de estos. Se pusieron entre 12 y 14 claves (distintos métodos) a disposición de los servicios estatales de salud, de acuerdo con un ejercicio de planeación en el que se consideró la población por proteger y las unidades de salud donde debían ser distribuidos, entre otros elementos. Se adquirieron desde condones hasta métodos de anticoncepción reversible de acción prolongada (por ejemplo, implantes).

\section{Focalización}

Las 32 entidades federativas priorizaron las estrategias de prevención del embarazo en niñas y adolescentes en 200 municipios y 135 jurisdicciones sanitarias, que integraban $56.3 \%$ de las mujeres de 15 a 19 años y cubrían $54.1 \%$ del total de los nacidos vivos de madres de 10 a 14 años de edad y $57.9 \%$ de los nacidos vivos de madres de 15 a 19 años; $89 \%$ de los 200 municipios prioritarios contaban con al menos un Servicio Amigable para Adolescentes.

\section{Conclusiones}

En México, en el embarazo adolescente confluyen el bono demográfico perdido para un país, ${ }^{36}$ así como las consecuencias metabólicas, neurocognitivas y sociales a largo plazo que se deben documentar. Resulta obligado y urgente diseñar intervenciones educativas en salud y normas culturales con énfasis en el plan de vida de las niñas adolescentes. Dichas intervenciones deben ser tempranas, evaluadas, replicables y sustentables en todo el país. El embarazo adolescente continúa siendo uno de los principales factores que contribuyen a la mortalidad materno-infantil y a perpetuar el círculo de enfermedad y pobreza. No incidir en esta problemática es apuntalar una forma de pobreza planificada. ${ }^{37}$

Ha habido respuesta institucional que debe ser evaluada y, de ser necesario, modificada. Se han obtenido algunos logros alentadores, si bien insuficientes. Es deseable que se continúe con aquello que después de ser evaluado muestre una contribución positiva para enfrentar el problema y se modifique 0 elimine lo que no tenga un impacto en la disminución de los embarazos adolescentes.

\section{Bibliografía}

1. Adolescent Pregnancy. Suiza: World Health Organization; 2018

2. El Banco Mundial [sitio web]. México: Evaluación del programa de país: Evaluación del apoyo del Grupo del Banco Mundial a México (2008-17): Panorama General. Independent Evaluation Group. EE. UU.: World Bank Group; 2018.

3. World Health Organization [sitio web]. Safe abortion: technical \& policy guidance for health systems. Legal and policy considerations: laws and policies on abortion should protect women's health and their human rights. Suiza: World Health Organization; 2015.

4. El cumplimiento de la agenda 2030 y los objetivos de desarrollo sostenible en México. México: Gobierno de la República; 2017.

5. Comisión Económica para América Latina y el Caribe. Consenso de Montevideo sobre población y desarrollo: Cepal; 20138.

6. Consejo Nacional de Población. Proyecciones de la población de México y de las entidades federativas 2016-2050. México: Conapo; 2018.

7. Consejo Nacional de Población. Seguimiento del Grupo Interinstitucional para la Prevención del Embarazo en Adolescentes (GIPEA). México: Conapo; 2018.

8. Grupo Interinstitucional para la Prevención del Embarazo en Adolescentes. Estrategia Nacional para la Prevención del Embarazo en Adolescentes (ENAPEA). Informe 2016. México: 2016.

9. Jiménez GA, Granados CJA, Rosales FRA. Embarazo en adolescentes de una comunidad rural de alta marginalidad. Un estudio mixto de caso. Salud Publica Mex. 2017;59:11.

10. Grupo Interinstitucional para la Prevención del Embarazo en Adolescentes. Estrategia Nacional para la Prevención del Embarazo en Adolescentes (ENAPEA). Informe 2018; México: 2018.

11. Instituto Nacional de Estadística y Geografía. Estadísticas a propósito del día de la madre. México: INEGI; 2018.

12. Hall AM, Kutler BA. Intrauterine contraception in nulliparous women: a prospective survey. J Fam Plann Reprod Health Care. 2015;42:36-42.

13. González E, Leal I, Molina T, Chacón P. Patrón intergeneracional de embarazo adolescente en las hijas de una cohorte de mujeres que controlaron su primer embarazo en un centro integral para adolescentes embarazadas. Rev Chil Obstet Ginecol. 2013;78:282-289.

14. Gupta J, Falb KL, Ponta O, Xuan Z, Campos PA, Gómez AA, et al. A nurse-delivered, clinic-based intervention to address intimate partner violence among low-income women in Mexico City: findings from a cluster randomized controlled trial. BMC Med. 2017:15:128.

15. Organización Mundial de la Salud. Comprender y abordar la violencia contra las mujeres. Suiza: OMS; 2018.

16. Motherhood in childhood. Facing the challenge of adolescent pregnancy. United Nations Population Fund: EE. UU.: 2013

17. Instituto Nacional de Estadística y Geografía. Muerte materna y muertes evitables en exceso. México: INEGl; 2014.

18. Papathakis $P C$, Singh $L N$, Manary MJ. How maternal malnutrition affects linear growth and development in the offspring. Mol Cell Endocrinol. 2016;435:40-47.

19. Karataşlı V, Kanmaz AG, İnan AH, Budak A, Beyan E. Maternal and neonatal outcomes of adolescent pregnancy. J Gynecol Obstet Hum Reprod. 2019:48:347-350.

20. Zabludovsky G. Las mujeres en los ámbitos de poder económico y político de México. Rev Mex Cienc Polit Soc. 2015;250(223):61-94. 
21. Navarro DM, Narro-Robles J, Orozco-Hernández L. La mujer en México: inequidad, pobreza y violencia. Rev Mex Cienc Polit Soc. 2014;59:117-147.

22. Spies Shapiro LA, Margolin G. Growing up wired: social networking sites and adolescent psychosocial development. Clin Child Fam Psychol Rev. 2014;17:1-18.

23. Blancarte R. Religión y sociología; cuatro décadas alrededor del concepto de secularización. Estudios Sociológicos. 2012;30:59-81.

24. Norma oficial mexicana NOM-046-SSA2-2005, violencia familiar, sexual y contra las mujeres. Criterios para la prevención y atención. México: Diario Oficial de la Federación; 2009 Mar 24.

25. Norma oficial mexicana NOM 005-SSA2-1993, de los servicios de planificación familiar. México: Diario Oficial de la Federación 1994 May 30.

26. Norma oficial mexicana NOM-047-SSA2-2015, para la atención a la salud del grupo etario de 10 a 19 años de edad. México: Diario Oficial de la Federación; 2015 Ago 12

27. Flores-Pérez E, Amuchástegui-Herrera A. Interrupción legal del embarazo: reescribiendo la experiencia del aborto en los hospitales públicos del Distrito Federal. Género y Salud en Cifras. 2012;10:21-30.

28. World Health Organization Safe abortion: technical and policy guidance for health systems. Suiza: WHO; 2012

29. The world's abortion laws 2014. EE. UU.: Center for Reproductive Rights; 2014.
30. Sámano $R$, Martínez-Rojano $H$, Robichaux D, Rodríguez-Ventura AL, Sánchez-Jiménez B, Segovia S, et al. Family context and individual situation of teens before, during and after pregnancy in Mexico City. BMC Pregnancy Childbirth. 2017; 17:382.

31. Chang T, Choi H, Richardson CR, Davis MM. Implications of teen birth for overweight and obesity in adulthood. Am J Obstet Gynecol. 2013;209:110-117.

32. World Health Organization Preventing early pregnancy and poor reproductive health outcomes among adolescents in developing countries. Suiza: WHO; 2011.

33. Instituto Nacional de Estadística y Geografía. Estadísticas vitales. Natalidad 2017. México: INEGI; 2018.

34. Organización Mundial de la Salud. Salud de la madre, el recién nacido, del niño y del adolescente. Suiza: OMS; 2017.

35. Dirección General de Información en Salud Servicios otorgados SIS. Cubos dinámicos. México: Secretaría de Salud; 2019.

36. Amjad S. MacDonald I, OsornionVargas A. Social determinants of health and adverse maternal and birth outcomes in adolescent pregnancies: a systematic review and meta-analysis. Paediatr Perinat Epidemiol. 2019;33:88-99.

37. Bruce J. The difficulties of 'living while girl'. J Virus Erad. 2016;2: 177-182. 